\title{
THEORETICAL STUDIES OF DENSE CLOUD CHEMISTRY
}

\author{
Eric Herbst \\ Department of Physics \\ Duke University \\ Durham, NC 27706 \\ USA
}

\begin{abstract}
Based on analyses by a variety of investigators, it has become understood that gas phase reactions can account for much of the chemistry observed in dense interstellar clouds. However, quantitative calculations of molecular abundances utilizing gas phase reactions are beset with difficulties. These difficulties include uncertainties in needed rate coefficients at the low temperatures of interstellar clouds, uncertainties in the dynamics of physical processes such as cloud collapse and clumping, and uncertainties in our understanding of gasgrain interactions. New work in some of these areas and its impact on modelling is emphasized.
\end{abstract}

\section{INTRODU CTION}

In the last international meeting on astrochemistry, held in Bad Windsheim, FRG during the summer of 1984, I reviewed the status of gas phase chemical models of dense interstellar clouds with an emphasis on the production of complex molecules (Herbst 1985a). In that review, I first summarized the accomplishments of these models, specifically the prediction of molecular abundances in reasonable agreement with observation for many species. I then emphasized that much more work remained to remove deficiencies in the models. In this review, I shall discuss work that has occurred in the last year and a half which relates to improving the deficiencies I mentioned. The major physical and chemical details underlying gas phase models are contained in a new review article (Winnewisser and Herbst 1985).

The basic deficiencies in gas phase chemical models are as follows:

(i) Almost all models utilize fixed, homogeneous physical conditions whereas it is known that dense clouds are dynamic and, to a varying degree, heterogeneous. Even within the framework of fixed physical conditions, it is uncertain whether abundances calculated at steady state or at earlier times when molecular abundances, especially those of complex molecules, are generally larger, are more applicable. 
(ii) Models do not typically include gas-grain interactions such as adsorption and desorption.

(iii) Models utilize rate coefficients that are of ten estimated or based on experimental results at $300 \mathrm{~K}$ rather than at lower temperatures.

(iv) Models tend to neglect photoprocesses, which are clearly important in dense cloud edges, and may even be important in cores of small, dark clouds.

(v) Models are sensitive to undetermined initial conditions such as gas phase elemental abundances.

(vi) Models have not yet included the most complex interstellar molecules observed nor made predictions on the ultimate complexity attainable by gas phase chemistry.

These deficiencies and current work to alleviate them will be discussed in turn.

\section{RECENT WORK}

\subsection{Physical Conditions and Time Dependence}

Even in models that do not consider any change in physical conditions with time, the time dependence of the chemical species can be complex. In the model of Leung et al. (1984), most large molecules are produced at very high abundances at times well before steady state is achieved. These abundances then decrease with time until steady state is reached ( $t \geq 10(7) \mathrm{yr}$.$) , at which time calculated abundances are well below$ observed values. This time dependence, which is ascribed to the need for complex molecules to " $f$ ix" atomic carbon, itself a species calculated to be in high abundance only at intermediate times, is not obtained for many of the simpler molecules in the model. The observation of copious amounts of atomic carbon in selected dense clouds suggests that perhaps we are observing clouds that have not yet reached steady state. However, this conclusion is suspect because the model does not allow physical conditions such as density and temperature to change. In addition, a more recent model by Millar and Nejad (1985) contains only qualitative agreement with Leung et al. (1984) and shows much better agreement with observation at steady-state for complex molecular abundances.

Inclusion of physical time dependence is at the heart of the evolutionary model of Tarafdar et al. (1985) which considers the effect on the interstellar chemistry of simple molecules of the dynamical evolution of an initially diffuse, quiescent cloud into a dense cloud. Leung and Herbst (1985) are currently undertaking model calculations that include complex molecule chemistry and that treat cloud collapse, albeit in a far more simple manner than do Tarafdar et al. (1985). 


\subsection{Gas-Grain Interactions}

In models that do not consider cloud evolution and collapse, a natural barrier to the achievement of steady-state abundances is the probability that molecules adsorb onto grain surfaces on a rather short time scale. Based on diffuse cloud observations, one can estimate an adsorption time of $3 \times 10(9) / n \mathrm{yr}$. where $\mathrm{n}$ is the total gas density. This unphysically small number is not decisively changed by coagulation of grains in dense clouds (Draine 1985). The adsorption time is necessarily increased by evolutionary models that utilize initial conditions with low gas densities. However, the short adsorption time noted here for constant $n$ can be effectively lengthened by desorption processes involving cosmic ray-induced heating, as has been discussed recently by Leger et al. (1985). Whether or not such desorption processes merely lengthen the time scale for $100 \%$ absorption or result in a true steady-state condition is not yet understood.

\subsection{Rate Coefficients}

In the last year to year and a half, much theoretical and laboratory work has occurred in the field of ion-molecule reactions. Perhaps the major new theoretical advance has been the "AC" theory of Clary (Clary 1985), which is related to earlier work of Sakimoto (1981) and

Takayanagi (1978). Results using the "AC" theory suggest that reactions between ions and polar neutrals are 1-2 orders of magnitude faster at temperatures near $10 \mathrm{~K}$ than is predicted by simple models such as the Langevin or ADO. Other theories of ion-polar neutral reaction rates yield somewhat smaller but still large values for rate coefficients at $10 \mathrm{~K}$ (Bates 1982). Adams, Smith, and Clary (1985) have presented some experimental evidence favorable to the "AC" theory for reactions between ions and linear polar neutrals and have calculated several very large rate coefficients at interstellar temperatures for reaction systems of this type. Millar et al. (1985) have shown that the use of large rate coefficients for the depletion reactions of CS, a very polar species, explains the heretofore poorly understood [HCS+]/[CS] interstellar abundance ratio. I (Herbst $1985 \mathrm{~b}$ ) have advocated some caution in adopting large rate coefficients for reactions that have been measured to be especially slow at room temperature.

To determine the effect on large scale models of larger rate coefficients for ion-polar neutral species, Leung and $I$ are using two theoretical treatments simpler than the "AC" theory, but more practical considering the large number of reactions involved. For reactions involving linear polar molecules, we are utilizing the "locked dipole" treatment which is an adequate representation of reality for low temperature, low density regions where linear polar neutrals are primarily relaxed into low-lying rotational states. For reactions involving non-linear polar molecules, which have a number of metastable rotational states and are therefore more complex to treat, we are utilizing the treatment of Sakimoto (1985), which is based on a formula of Su and Chenavich (1982). Use of this formula results in smaller rate 
coefficients for the non-linear case than for the linear case, although both cases show an inverse temperature dependence that is approximately an inverse square root one. Most recently, Rowe (1985) has measured some ion-molecule reactions involving the non-linear reactants water and ammonia at $28 \mathrm{~K}$ and found reaction rate coefficients significantly larger than at room temperature and in reasonable if not excellent agreement with the Su and Chesnavich (1982) formula. More rate measurements at this low temperature would be a real boon to interstellar astrochemistry!

Use of these large ion-polar neutral rate coefficients in our model results in the following salient changes at $10 \mathrm{~K}$. The peak abundances of highly polar neutrals such as $\mathrm{HC}_{3} \mathrm{~N}$ occur at even earlier times and are reduced by a factor of 3-10 from previous model results. The unusual time dependence observed by Leung et al. (1985) for complex species is preserved. Steady-state abundances of polar neutrals are also lowered by a factor of 3-10. Abundances of ions produced from polar neutrals (e.g. $\mathrm{HCS}^{+}$) are enhanced. The fractional ionic abundance is changed only slightly.

In the area of complex molecule synthesis, Federer et al. (1985) have just completed a very useful study of ion-molecule reactions involving hydrocarbon ions and atomic nitrogen. Several authors including myself had previously suggested these reactions to be the most efficient routes for cyanoacetylene synthesis. For example, consider the reaction

$$
\mathrm{C}_{3} \mathrm{H}_{3}^{+}+\mathrm{N}---\rightarrow \mathrm{H}_{2} \mathrm{C}_{3} \mathrm{~N}^{+}+\mathrm{H}
$$

which leads to a precursor ion to $\mathrm{HC}_{3} \mathrm{~N}$. Federer et al. (1985) have shown this and similar reactions to occur in the laboratory despite the frequent necessity of flipping electronic spins. However, these reactions do not seem to occur for larger hydrocarbon ions such as those with four carbon atoms, perhaps implying that this mechanism cannot be expected to synthesize the larger cyanoacetylenes in interstellar clouds.

I have continued my research into ion-molecule radiative association reactions. These processes, in which an ion and a neutral collide to form a short-lived "collision complex" which stabilizes itself via emission of a photon, are difficult to study in the laboratory yet are most important in the gas phase syntheses of complex molecules in interstellar clouds (Leung et al. 1984). In a recent work, I have recalculated the rate coefficients for a significant number of these reactions utilizing new information on spontaneous emission rates of molecular ions (Herbst 1985c; see also Bates 1985). The new calculated rates are larger by about an order of magnitude than the old. I have also investigated the possibility that radiative association channels can compete with normal ion-molecule reaction channels at low temperature under certain circumstances (Herbst 1985d) and have illustrated these circumstances with a calculation on the reactions between ammonia and the methyl ion. The ability of radiative association processes to compete with normal exothermic channels is necessary in the suggested syntheses of a variety of complex 
interstellar molecules (Huntress and Mitchel1 1979). Calculations on additional systems are being undertaken.

In another calculation on radiative association rate coefficients, I have shown how to calculate these rates under the non-equilibrium conditions present in shocked interstellar gas (Herbst 1985e) and have illustrated the theory with a calculation on the association between methyl ions and molecular hydrogen. A principal result of this theory is that the known inverse temperature dependence of radiative association rate coefficients is not as severe as suggested by theoretical studies in which non-equilibrium effects are neglected.

There are several areas in the study of rate coefficients which have not yet been approached successfully from either laboratory experiment or theory and these deserve special mention. First is the question of the identity of the neutral products of ion-electron dissociative recombination reactions. These reactions have been studied in the laboratory and their rate coefficients measured but, to the best of my knowledge, only one experimental study of the product branching ratio has appeared. It is critical that these products be determined. Up to now, most modelers have assumed that when a large hydrogencontaining ion combines dissociatively with an electron, only a hydrogen atom or molecule is split of from the neutral skeletal framework; viz.,

$$
\mathrm{H}_{2} \mathrm{C}_{3} \mathrm{~N}^{+}+\mathrm{e}---\mathrm{HC}_{3} \mathrm{~N}+\mathrm{H} ; \mathrm{C}_{3} \mathrm{~N}+\mathrm{H}_{2} \text {. }
$$

The possibility exists, however, that the neutral molecule can be more severely disrupted than by simple hydrogen elimination. If ion-electron dissociative recombination reactions do result preferentially in severely fragmented neutrals, then the synthetic power of ion-molecule reaction networks will be severely curtailed.

Another area in dire need of experimental investigation is low temperature neutral-neutral reactions. Many reactions of this type involving either atoms or radicals do not appear to have activation energy and are therefore of some importance in gas phase chemical models of dense clouds. However, few have been studied at temperatures anywhere near that of interstellar clouds and the possibility of small barriers cannot be overlooked. Consider, as an example, the reaction

$$
\mathrm{C}+\mathrm{O}_{2}-\cdots \mathrm{CO}+\mathrm{O}
$$

which is normally assumed to be rapid at low temperatures based upon some much higher temperature laboratory work. If this reaction were unexpectedly slow at low temperatures, then the $\mathrm{C} / \mathrm{CO}$ balance in dense clouds would be markedly changed in favor of more $C$ and less $\mathrm{CO}$, a direction which might bring model results at steady state closer to observational reality.

\subsection{Photoprocesses}

The effect of photodestruction processes on standard ion-molecule chemistry involving polyatomic species has been little explored, 
although Tielens and Hollenbach (1985) have recently explored in some detail the situation as regards smaller species. Herbst and Leung (1985) have recently attempted to repair this neglect by tabulating photodestruction rate coefficients for many polyatomic neutrals as functions of visual extinction. They have utilized these rates in two model calculations with fixed physical conditions - one of the core of a compact dense cloud with a visual extinction of 5 , and one of a diffuseto-dense cloud (gas density of $2 \times 10(3)$ per $c c, T=50 \mathrm{~K}$ ) with a visual extinction of 2. This latter model is a reasonable representation of regions of dense clouds away from central condensations. In agreement with Millar and Freeman (1984), the former model indicates that photoprocesses are not very important in cloud cores even if the visual extinction is as low as five. In the latter model, steady state is reached in a rather short time and the maximum abundances of complex molecules are achieved at steady state. The fractional electron abundance of $2 \times 10(-5)$ is much higher than in dense cloud cores and little complex molecule development occurs, except perhaps in the bare hydrocarbons. A high CO fractional abundance of $7.7 \times 10(-5)$ is calculated, in agreement with observed large abundances of $\mathrm{co}$ outside of cloud core regions. This model also appears to explain the high $\mathrm{CH}$ abundance observed by Ziurys and Turner (1985) and the $\mathrm{HOC}^{+} / \mathrm{HCO}^{+}$ratio observed by Woods et al. (1983; see Jarrold et al. 1985 for a complete discussion.)

\subsection{Sensitivity}

Gas phase models are sensitive to the elemental abundances utilized. Although this sensitivity has not been investigated thoroughly for all the dominant elements, several studies have been done on the $\mathrm{C} / 0$ abundance ratio. A $\mathrm{C} / 0$ ratio greater than unity was suggested by Langer et al. (1984) as one possible explanation of the observed large C I abundance in dense clouds. Model calculations using a large $\mathrm{C} / 0$ ratio of 1.28 rather than the more normal value of less than unity have been performed by Langer et al. (1984) and Herbst and Leung (1985), both of whom increased the size of the $C$ abundance and maintained a constant 0 abundance. Watt (1985) has presented model calculations for thirty simple species as a function of $\mathrm{C} / 0$ ratio from 0.1 to 2.0 , in which the 0 abundance is varied. All models agree in their prediction of large amounts of C I at steady state, in agreement with observation. In other calculated abundances, there are disagreements, even between the results of Langer et al. (1984) and Herbst and Leung (1985). In our model, the time dependence of complex molecule abundances, discussed in Leung et a1. (1984), is not seen for non-oxygen-containing species. Steady-state abundances of these species are considerably greater than what is observed in dense clouds, possibly because the $C$ abundance is set at an unrealistically high level. 


\subsection{More Complex Molecules}

Little progress in including complex molecules into models has occurred recently. Even the models of Leung et al. (1984) and Herbst and Leung (1985) contain only a representative variety of complex molecules. Inclusion of a wider variety of complex molecules such as all the observed cyanoacetylenes is necessary so that detailed comparisons with observation can be made. The problem up to now has been the lack of experimental rate coefficients on important reactions such as hydrocarbon ion- $\mathrm{H}_{2}$ reactions and condensation reactions.

\section{OTHER STUDIES}

In this section, I would like to discuss briefly some important work on isotopic fractionation and metastable isomers that has appeared recently. Studies of this sort calculate abundance ratios of similar species rather than actual abundances and are less affected by timedependent considerations. Two major works on deuterium fractionation have appeared recently that emphasize the importance of $D$ atoms (Dalgarno and Lepp 1984; Croswell and Dalgarno 1985). For example, the production of $O D$ in diffuse clouds occurs primarily through the reaction

$$
\mathrm{D}+\mathrm{OH} \longrightarrow-->\mathrm{OD}+\mathrm{H}
$$

whereas the reverse reaction, endothermic by $810 \mathrm{~K}$, is not important in cold clouds. Enhancements for $\mathrm{OD} / \mathrm{OH}$ relative to $\mathrm{D} / \mathrm{H}$ of $30-400$ are found in diffuse clouds with even greater enhancements in dense clouds.

Two recent studies of abundance ratios between metastable and stable isomers have appeared. DeFrees et al. (1985) have calculated the $\mathrm{CH}_{3} \mathrm{NC} / \mathrm{CH}_{3} \mathrm{CN}$ abundance ratio to be approximately 0.1 , in good agreement with observation. Jarrold et al. (1985) have calculated the $\mathrm{HOC}^{+} / \mathrm{HCO}^{\mp}$ abundance ratio in a variety of dense and diffuse cloud models and find that the observed value of $1 / 330$ can only be $f$ it by diffuse or near diffuse cloud models; The basic problem in dense clouds is that there is evidence that $\mathrm{HOC}^{+}$reacts rapidly with $\mathrm{H}_{2}$ at low interstellar temperatures.

\section{FUTURE WORK}

Directions for future work in modeling and other studies have already been touched on above. It is my view that a large proportion of future work will be directed towards an appreciation of the diversity of chemistry in regions of different physical conditions from the normal dense cloud environment such as cloud edges, hotter regions around protostars, shocked regions, and active maser regions. Studies of chemical diversity involving small regions will most certainly become even more important as millimeter interferometers yield an increasing richness of results. 


\section{ACKNOWLEDG EMENTS}

I would like to thank the National Science Foundation (U.S.) for support of my research program in astrochemistry through grant AST - 8312270 .

\section{REFERENCES}

Adams, N.G., Smith D. \& Clary, D. C. 1985, Ap. J. (Letters) 296, L31. Bates, D. 1982 , Proc. R. Soc. Lond. A 384, 289.

Bates, D. 1985, Ap. J. 1985, 298, 382 .

C1ary, D. C. 1985, Molec. Phys. 54, 605.

Croswel1, K. \& Dalgarno, A. 1985, Ap. J. 289, 618.

Dalgarno, A. \& Lepp, S. 1984, Ap. J. (Letters) 287, 147.

DeFrees, D. J., McLean, A. D. \& Herbst, E. 1985, Ap. J. 293, 236.

Draine, B. 1985, in Protostars \& Planets II (Tucson: U of Arizona)

Federer, W. et al. 1985, submitted to Chem. Phy s. Letters.

Herbst, E. 1985a, in Molecular Astrophysics - State of the Art and

Future Directions (Dordrecht:Reidel).

Herbst, E. 1985b, submitted to Ap. J.

Herbst, E. 1985 c, Ap. J. 291, 226.

Herbst, E. 1985 d, Ap. J. 293, 236.

Herbst, E. 1985 e, Astron. Astrophys., in press.

Herbst, E. \& Leung, C. M. 1985, submitted to Mon. Not. R. Astr. Soc.

Huntress, W. T., Jr. \& Mitche11, G. F. 1979 , Ap. J. 231, 456.

Jarrold, M. F. et a1. 1985, Ap. J., in press.

Langer, W. D. et al. 1984, Ap. J. 277, 581 .

Leger, A., Jura, M. \& Omont, A. 1985, Astron. Astrophys. 144, 147.

Leung, C. M., Herbst, E. \& Huebner, W. F. 1984, Ap. J. Suppl. 56, 231.

Millar, T. J., Adams, N. G., Smith, D., and Clary, D. C. 1985, Mon. Not. R. Astr. Soc. 216, 1025.

Millar, T. J. \& Freeman, A. 1984, Mon. Not. R. Astr. Soc. 207, 405.

Millar, T. J. \& Nejad, L. A. M. 1985, Mon. Not. R. Astr. Soc., in press.

Rowe, B. R. 1985, private communication as reported by E. E. Ferguson.

Sakimoto, K. 1981, Chem. Phy s. 63, 419.

Sakimoto, K. 1985, Chem. Phys. Letters 116, 86.

Su, T. \& Chesnavich, W. J. 1982 , J. Chem. Phy s. 76, 5183.

Takay anagi, K. 1978 , J. Phys. Soc. Japan 45, 976.

Tarafdar,'S. P. et al. 1985, Ap. J. 289, 220.

Tielens, A. G. G. M. \& Hollenbach, D. 1985, Ap. J. 291, 722 .

Watt, G. D. 1985, Mon. Not. R. Astr. Soc. 212, 93.

Winnewisser, G. \& Herbst, E. 1985, "New Organic Molecules in Space', in Topics in Current Chemistry (Berlin, Springer Verlag, in press)

Woods, R. C. et al. 1983, Ap. J. 270, 583 .

Ziurys, L. M. \& Turner, B. E. 1985, Ap. J. (Letters) 292, L25. 
DISCUSSION

TARAFDAR: Dr. Ohishi showed that so and $\mathrm{SO}_{2}$ molecules are enhanced in outflow regions. Can you explain how they are produced?

HERBST: I do not want to comment on shock models as they will be discussed by persons more qualified than me.

TATUM: All of the observed polyynes have odd numbers of carbon atoms. Does this mean that polyynes with even numbers of carbon atoms are absent, or does it merely mean that such molecules would have to have a hydrogen (or a nitrogen) at each end, and hence would be unobservable owing to lack of dipole moment?

HERBST: Polyynes with an even number of carbon atoms (e.g. HCCN) have not been studied extensively in the laboratory.

SAXENA: (i) What is the major source of $\mathrm{C}^{+}$in Orion $\mathrm{A}$, and (ii) source of ionization, other than cosmic ray, which is of importance for Orion A?

HERBST: (i) At steady state, $\mathrm{C}^{+}$derives from

Cosmic Rays $+\mathrm{He} \rightarrow \mathrm{He}^{+}+\mathrm{e}+$ Cosmic Rays, and from $\mathrm{CO}+\mathrm{He}^{+} \rightarrow \mathrm{C}^{+}+\mathrm{O}+\mathrm{He}$. (ii) For large $A_{V}$, cosmic ray ionization dominates.

GREENBERG: I have my usual question of "how can you reconcile gas phase chemistry alone if the time scale for grain accretion at $\mathrm{n}_{\mathrm{H}}=10^{4} \mathrm{~cm}^{-3}$ is $\tau_{\mathrm{ac}} \cong 3 \times 10^{5} \mathrm{yr}$ which is significantly shorter than the chemical time scales?" If one requires the grain molecules to be desorbed then, I believe, the mechanism of desorption must play a role in the chemistry in addition to whatever surface reactions take place. HERBST: The time scale for gas phase chemistry is comparable to the adsorption time in the sense that significant abundances are achieved in $3 \times 10^{5} \mathrm{yr}$. However, desorption mechanisms are clearly important if near steady-state abundances are to be achieved.

D'HENDECOURT: Because the accretion time scale on grains is so short, is it not true that every detected molecule in a dense cloud $\left(n_{\mathrm{H}}>10^{3} \mathrm{~cm}^{-3}\right.$ ) has encountered a grain one or two (or more) times in the lifetime of the cloud?

HERBST: So it would appear.

VAN DISHOECK: Could you comment on the large differences that are found in the predicted abundances of various species in your models and, for example, those of Millar et al.?

HERBST: The calculation of Millar and Nejad (1985) has slightly different elemental abundances from ours and does not contain reactions involving hydrocarbon ions and 0 atoms. In our model, these reactions are important inhibitors to complex molecule development. 
PIRRONELIO: Could you please comment on the $\mathrm{H}_{2}$ formation problem, especially in the light of Smoluchowski results which show an extremely low mobility of $\mathrm{H}$ atoms on amorphous grains?

HERBST: I am not expert on this problem.

K.K. GHOSH: What are the important ions that play the dominant role for ambipolar diffusion in interstellar dark clouds during the stage of contraction?

HERBST: The dominant ions are metal ions and the molecular ions $\mathrm{HCO}^{+}$ and $\mathrm{H}_{3}^{+}$. 